\title{
Dialectical Thinking and Method to Cultivate Student Creativity in the Teaching of Probability Statistics
}

\author{
Xiao Xiaonan \\ School of Information Science \& Technology \\ Tan Kah Kee College of Xiamen University \\ Zhangzhou, Fujian, China \\ xiaoxn@xujc.com
}

\begin{abstract}
Through my discussion about dialectical thinking of inferential statistics in the teaching of probability statistics and the analysis of the related difficult issues, this article explores in depth the dialectical method in college maths teaching and the dialectical relations between teaching and scientific research. It satisfactorily builds the connections and dialogues between interval estimation and hypothesis testing in the teaching of statistical inference. It improves students' knowledge and comprehension of this difficult part in teaching, and cultivates and expands students' creative thinking and ability to study independently and innovatively.
\end{abstract}

Keywords-Probability statistics; Statistical inference; dialectical thinking; Interval estimation; Hypothesis testing; Creative thinking

\section{FURTHER INNOVATING AND REFORMING TEACHING; CULTIVATING CREATIVE HIGH-QUALITY TALENTS}

Probability theory and mathematical statistics are maths disciplines that study stochastic phenomenon from the aspect of quality. With the advent of knowledge economy and informational era, maths theories and methods that study stochastic phenomenon and their application spread out and become prevalent. The methodology of probability theory has penetrated into all areas of natural science and social science and continues expanding.

Probability theory and mathematical statistics is not only an important basic course in the field of engineering and economics but also a crucial compulsory subject of the national entrance examination of the post graduate program, which has been for years listed as one of the core courses for assessment by the National Ministry of Education.

The rapid development of modern science and technology has accelerated the updating of knowledge. Therefore, it has become essential that we explore new ways and adjust the teaching of Probability Statistics, an important basic course of science, engineering and economics, to meet the requirement of the development of knowledge and economics. In order to improve teaching quality of Probability Statistics and to meet the need for interdisciplinary, high-quality talents in science, engineering, and economics in the new century, we must continue teaching reform and innovation and train creative high-quality talents who can adjust to the information age.
The teaching objective of Probability Theory and Mathematical Statistics is to train students to apply the theory, methods and techniques learned in the course to fully analyze and solve various problems in the study of natural science, engineering, and economics. How students have learned in Probability Statistics not only directly affect many other following courses in the future but also on their entrance exam of post-graduate program. However, since Probability Theory and Mathematical Statistics are different from Advanced Mathematics in its way of thinking [1], and moreover the content of stochastic mathematics is rather rich, abstract, and complex in its approach of tackling problems, which is entitled to high-level comprehension and skill [2], the beginning learners are struggling and craving for a teaching method and mode that is more relevant, practical, easy to understand, focused, and innovative [3-4]. explains profound theories in simple language, makes the focal points stand out, scatters the hard points and is full of creativity. Thus we have explored teaching methods to greatly improve students' maths quality and innovative ideas for the teaching reform of Probability Statistics when we build the provincial quality course of Probability Statistics [5]. Through my discussion about dialectical thinking of inferential statistics in the teaching of probability statistics and the analysis of the related difficult issues, this article explores in depth the dialectical method in college maths teaching and the dialectical relations between teaching and scientific research [6]. It furthermore incites students' intellectual curiosity, cultivates their creativity, and expands their creative thinking and ability to study independently and innovatively.

\section{THE NEW IDEAS OF DIALECTICAL THINKING IN HYPOTHESIS TESTING TEACHING DIALECTICAL}

The basic problems of statistical inference in the teaching of probability statistics can be divided into two categories: one is the parameter estimation problem, the other is hypothesis testing. In the current textbooks, discussions of hypothesis testing problems do not demonstrate its relation with the estimated range, but only discuss the issue separately. However, the mathematical concepts have a deeper internal relationship between each other, although they may seem unrelated on the surface. It is the same case with hypothesis testing and interval estimation [7]. The author have been exploring the connection between interval estimation and hypothesis testing in his many 
years of teaching and research, and therefore found new ideas of hypothesis testing [8].

\section{THE INTERNAL RELATIONS AND DISTINCTIONS BETWEEN} INTERVAL PARAMETER ESTIMATION AND HYPOTHESIS TESTING

Although interval parameter estimation and interval hypothesis testing have different names, their approaches to solve the problem is related. The methodologies of statistic inference is the same, which is to select a statistic and then make the probability of this statistic fall on a small known range (equal to the known $\alpha$ ). Hence obtaining the results.

For example, the variance $\sigma^{2}$ is unknown, on the interval estimation of the mean $\mu$ for normal population $X$ and the testing hypothesis of $H_{0}: \mu=\mu_{0}$, we select statistic

$$
T=\frac{\bar{x}-\mu}{s / \sqrt{n}} \sim t(n-1)
$$

for a given $\alpha$, we get $t_{\alpha / 2}(n-1)$ so that

$$
P\left\{|T|>t_{\alpha / 2}(n-1)\right\}=\alpha
$$

- if $H_{0}: \mu=\mu_{0}$ testing, then $|T|>t_{\alpha / 2}(n-1)$ is the rejection region $W$ (the $\alpha$ is significance level);

- if we do the interval estimation for $\mu$, then from (1) we have the formula

$$
P\left\{|T|<t_{\alpha / 2}(n-1)\right\}=1-\alpha
$$

we extract from the brackets of (2) and get $\mu$, therefore the $1-\alpha$ confidence interval for $\mu$ (1- $\alpha$ is confidence).

Thus, the process of interval parameter estimation and parameter statistical testing is related. But there are differences between the two: first, the requirements of the two vary. The interval estimation asks for the given range on the basis of a certain degree of confidence of unknown parameters, whereas hypothesis testing asks to judge the given value of the unknown parameters at a certain level. Second, the two have different levels of understanding of the problems. The former is does not concern the unknown parameters, while the latter concerns the unknown parameters, yet is not exactly sure about it.

Therefore, if we carry out a hypothesis testing on a known standard $\mu_{0}$, we can only consider interval parameter estimation if not given the standard range.

\section{THE FORMATION OF NEW IDEAS ON THE DIALECTICAL THINKING OF HYPOTHESIS TEST TEACHING}

If $\alpha(0<\alpha<1)$ is given, there is

$$
P\{\theta \in L\}=1-\alpha
$$

that is, $P\{\theta \in(R-L)\}=\alpha$
Here $R:(-\infty,+\infty)$ is called a confidence interval where a random interval $L$ is parameter $\theta$ and confidence is $1-\alpha$. Now we compare ( $0<\alpha<1$ is a small probability) in (3) and the true disposable formula in the significance test

$$
P\left\{\text { rejected } H_{0} \mid H_{0} \text { is true }\right\}=\alpha
$$

it is easy to find that if we want to testify $H_{0}: \theta=\theta_{0}$ through the significance level (probability of error) $\alpha$, we only need to see if there is $\theta_{0} \in L$. If $\theta_{0} \in L$, it should accept $H_{0}$. If $\theta_{0} \in(R-L)$, it should rejected $H_{0}$. This is because when interval $L$ can cover parameter $\theta$ whose probability is $1-\alpha$, i.e., when the probability of $\theta$ in the interval $R-L$ is $\alpha$, since the probability of

$$
P\{\theta \in(R-L)\}=\alpha
$$

is small, assuming $\theta_{0} \in(R-L)$, of course $\theta=\theta_{0}$ should be a small probability event. According to the small probability principle, small probability events can not happen in one-time sampling. That is the interval $R-L$ derived from one-time sampling is not going to include $\theta$ true value. So the $\theta_{0}$ value within $R-L$ true value range from $\theta$ is impossible. Therefore, we should reject the $\theta=\theta_{0}$ hypothesis.

From this perspective, interval estimation and hypothesis testing not only have a close and organic link between them, but also we can study the rejection region of hypothesis testing by using a confidence interval

\section{EXAMPLE DISCUSSION}

Supposing $X \sim N\left(\mu, \sigma^{2}\right), \sigma^{2}$ is generally known, $x_{1}, x_{2}, \cdots, x_{n}$ is a random sample from $X$ whose sample size is of $n$. Under the significance level $\alpha(0<\alpha<1)$ the hypothesis test is

$$
H_{0}: \mu=\mu_{0}, \quad H_{1}: \mu \neq \mu_{0}
$$

From (4), we get that if only we acquire the two-sided confidence interval $L$ out $\mu$ of the $1-\alpha$, then when $\mu_{0} \in(R-L)$, it reject $H_{0}$. Now drawing on $X \sim N\left(\mu, \sigma^{2}\right)$, we should select statistics

$$
U=\frac{\bar{X}-\mu}{a / \sqrt{n}} \sim N(0,1)
$$

( $\bar{X}$ is the sample mean), then based on the sub-site definition of standard normal distribution $\alpha$, we have

$$
P\left\{\frac{\bar{X}-\mu}{a / \sqrt{n}}<U_{\alpha / 2}\right\}=1-\alpha
$$

namely 


$$
P\left\{\frac{\bar{X}-\mu}{a / \sqrt{n}} \geq U_{\alpha / 2}\right\}=\alpha
$$

Moreover we find that the $1-\alpha$ confidence interval of $\mu$ is:

$$
L=\left(\bar{X}-\frac{\sigma}{\sqrt{n}} U_{\alpha / 2}, \bar{X}+\frac{\sigma}{\sqrt{n}} U_{\alpha / 2}\right)
$$

And the rejection region of $H_{0}$ is (the $\alpha$ significance level):

$$
R-L=\left(-\infty, \bar{X}-\frac{\sigma}{\sqrt{n}} U_{\alpha / 2}\right] \cup\left[\bar{X}+\frac{\sigma}{\sqrt{n}} U_{\alpha / 2},+\infty\right)
$$

Obviously, the (6) and the

$$
P\left\{\text { rejected } H_{0} \mid H_{0} \text { is true }\right\}=\alpha
$$

are equivalent.

Like the above double-sided test with respect to the mean $\mu$, it is not difficult to use the similar method with which we get the one-sided confidence of $1-\alpha$ for the $\mu$ to solve the $\mu$ rejection region issue for one-sided test [9-10].

\section{CONCLUSION}

This article uses interval estimation knowledge to solve the problem of rejection region in hypothesis test through the control of the probability of reducing error and getting the corrected answer and the neglect of the possible errors in the probability test. It inspires us. To accurately grasp the intrinsic link between the two mathematical concepts is the key to led to the new conclusion. And the organic link and distinction between interval estimation and hypothesis test affirm the dialectic relationship between mathematics.

\section{REFERENCES}

[1] Xiao Xiaonan. The optimal non-liner filtering and majorized algorithm of a kind of nonstationary stochastic transmission system [J]. Journal of Mathematical Study,2010,43(4): 342-351.

[2] He Weilian.Permanence for single species model with feedback control and finite continuous delays [J]. Journal of Fuzhou University (Natural science Edition), 2009,37(4):468-470.

[3] Xiao Xiaonan. Optimum operation and optimum analysis to permit coding function of a kind of signal transitive stochastic system [J]. Journal of Xiamen University Natural Science,2009,48(2):170-173.

[4] Shi Kaiquan, Yao Bingxue. Function S-rough sets and las identification [J]. Science in China Series F:Information Science,2008,51(5):499-510.

[5] Dogru O,Duman O.Statistical approximation of Meyer-Konig and Zeller operators based on q-integers [J]. Publ Math Debrecen,2006,68(12):199-214.

[6] Qiu J,Zhang L.F-intefere law genercetion and its feature recognition [J]. Journal of Systems Engineering and Electronics, 2009,20(4):777-783.

[7] Huang Tianyun. The restrained optimization pattern search method research progress [J]. Chinese Journal of Computers,2008,31(7):12001251.

[8] Lin R,Liu F.Fractional high order approximation methods for the nonlinear fractional ordinary differential equation[J].Nonlinear Analysis, 200766(4):856-869.

[9] Yan H,Wei Q L.Determining compromise weights for group decision making[J]. Journal of Operational Research Society,2002(53):680-687.

[10] Wei W L,Ysn H.A method of transferring polyhedron between the intersection-form and the sum-form[J].Computers and Mathematics with Applications,2001(41):1327-1342. 